\title{
A High-Resolution Large-Acceptance Analyzer for X-ray Fluorescence and Raman Spectroscopy
}

\author{
U. Bergmann, ${ }^{\mathrm{a}}$ and S. P. Cramer a,b
}

(a) Physical Biosciences Division, Lawrence Berkeley National Laboratory, Berkeley, CA 94720

(b) Department of Applied Science, University of California, Davis, CA 95616

\begin{abstract}
A newly designed multi-crystal X-ray spectrometer and its applications in the fields of X-ray fluorescence and X-ray Raman spectroscopy are described. The instrument is based on 8 spherically curved Si crystals, each with a 3.5 inch diameter form bent to a radius of $86 \mathrm{~cm}$. The crystals are individually aligned in the Rowland geometry capturing a total solid angle of $0.07 \mathrm{sr}$. The array is arranged in a way that energy scans can be performed by moving the whole instrument, rather than scanning each crystal by itself. At angles close to back scattering the energy resolution is between 0.3 and $1 \mathrm{eV}$ depending on the beam dimensions at the sample. The instrument is mainly designed for X-ray absorption and fluorescence spectroscopy of transition metals in dilute systems such as metalloproteins. First results of the Mn K $\beta$ (3p -> 1s) emission in photosystem II are shown. An independent application of the instrument is the technique of X-ray Raman spectroscopy which can address problems similar to those in traditional soft X-ray absorption spectroscopies, and initial results are presented.
\end{abstract}

Keywords: Bragg reflection, K-edge, Raman spectroscopy, Rowland circle, crystal optics, silicon, synchrotron radiation, transition metals, X-ray fluorescence, X-ray absorption

\section{INTRODUCTION}

Synchrotron radiation (SR), originally a parasitic by-product of high energy physics experiments and now a powerful research tool used in over 30 dedicated facilities word wide, is being applied very successfully to answer important questions in areas ranging from condensed matter physics to chemistry, structural biology, and other material earth and life sciences [1]. The spectrum of SR ranges from the low infrared up to hard X-rays, and insertion devices at second and third generation SR-facilities can produce very low divergent and intense SR beams with a brightness (\# of photons per second, solid angle, energy bandwidth and area) more than 10 orders of magnitude larger than that produced by conventional X-ray generators. Curved mirrors are used for focusing and devices, such as gratings, multilayers and Bragg crystals, are applied for the purpose of monochromatization and focusing. Bragg optics has indirectly profited from the rapidly growing computer industry, whose vast demand of large perfect crystal silicon has constantly driven the optimization of growths methods, and the instrument described in this work is an example of this symbiosis. (Today, perfect crystal silicon can be grown with up to 12 inch diameter at relatively low cost).

The motivation behind a high-resolution large-acceptance X-ray analyzer is its application to X-ray emission spectroscopy and the combination of X-ray absorption and emission spectroscopy [2,3]. The latter field is variously known as resonant X-ray emission (RXES) [4], resonant X-ray fluorescence (RXFS) [5], resonant X-ray inelastic scattering (RXIS, RIXS or REXS) [6,7] or X-ray resonant scattering or X-ray resonance Raman scattering (XRRS) $[8,9]$. The essential part of these experiments is high-resolution monochromatization of both the X-ray excitation beam and the scattered or emitted radiation. Since emission and scattering is essentially isotropic, the analyzer has to combine good energy resolution with large angular acceptance. The instrument described here has evolved from previous designs $[10,11]$ and its largely increased solid angle and efficiency makes it particularly suited for the study of very weak signals from, e.g., dilute biological samples and/or weak electromagnetic interactions. 


\section{BRAGG OPTICS}

The approximate energy of a Bragg reflection can be obtained from Bragg's law

$$
2 d \sin \theta=n \lambda,
$$

with the Bragg angle $\theta$, the integer $n$, the wavelength $\lambda$ and the $\mathrm{d}$-spacing $d=a /\left(h^{2}+k^{2}+l^{2}\right)^{1 / 2}$, where $a$ is the lattice parameter and $h, k, l$ are the Miller indices. Eq. (1) shows that the wavelength $\lambda$ has to be less than two times the crystal d-spacing, and therefore crystal monochromators are typically applied for energies well above $1 \mathrm{keV}$ (The conversion formula between wavelength $\lambda$ and energy $E$ is given by $E[\mathrm{keV}]=12.39852 /(\lambda[\mathbf{4}]))$. The angular width (often called Darwin width) and corresponding energy width of a perfect crystal Bragg reflection can be calculated with the dynamical theory [12-15], resulting in two simple rules. First, the relative energy resolution $\Delta E / E_{\mathrm{dyn}}$ is approximately constant for a given Bragg reflection, independent of the energy and the Bragg angle. Second, $\Delta E / E_{\mathrm{dyn}}$ decreases with higher order reflections. Furthermore, the derivative of Eq. 1 given by

$$
|\Delta E / E|=|\Delta \lambda / \lambda|=\Delta \theta \cot \theta
$$

shows that any angular divergence $\Delta \theta$ has the smallest contribution to the energy resolution $\Delta E / E$ at Bragg angles $\theta$ close to $90^{\circ}$. Hence, the best energy resolution of a perfect crystal monochromator can be obtained by using high order reflections at Bragg angles close to $90^{\circ}$. Besides the conceptually simpler single crystals (applied at almost exactly $90^{\circ}$ Bragg angle and therefore very limited in their scanning range) and nondispersive two crystal or channel cut crystal monochromators (typically applied to the white SR beam in a low order reflection), different types of high resolution devices can be used as either second stage monochromators, polarizers or analyzers. The design depends strongly on the nature of the radiation, and can be categorized in two different types, flat crystal and focusing devices.

Flat crystal monochromators are applied in the dispersive or $(+,+)$ geometry [16] and achieve essentially the intrinsic energy resolution $\Delta E / E_{\mathrm{dyn}}$, independent of the beam divergence. They have a relatively large scanning range and a good throughput for radiation with a low divergence comparable to the Darwin width of the Bragg reflection. Clearly such devices are not very efficient if the radiation is quasi isotropic, since they can only accept an extremely small fraction $\left(\square 10^{-8}\right)$ of the total $4 \pi$ sr solid angle.

To effectively monochromatize isotropic or quasi isotropic radiation emitted by a point like source, focusing optics in the Rowland geometry at Bragg angles close to $\theta=90^{\circ}$ (Eq. 2) can be applied. The focusing can be achieved by either spherically arranging a large number of small flat crystals, which, in fact, results in the best energy resolution, [17], or by bending a single crystal. In the Rowland geometry, ideally, each accepted ray impinges on the crystal surface under the same angle, and hence the energy resolution is given by the intrinsic crystal resolution. The accepted solid angle is determined by the size and radius of curvature of the crystal, and can be many orders of magnitude larger than in a $(+,+)$ geometry. Using several curved crystals together, the solid angle can be further increased without sacrificing energy resolution. Such an instrument and its applications are described below.

\section{THE INSTRUMENT}

\subsection{GEOMETRY AND ENERGY RESOLUTION}

An earlier design of a high-resolution X-ray fluorescence spectrometer working at $6.5 \mathrm{keV}$ is described by Stojanoff et al. [10] and the first multi crystal version is described by Wang et al. [11]. We have used a similar geometry, but have improved many details, most notably the solid angle, the efficiency and the scanning procedure. The spectrometer employs eight round Si crystals each with a diameter of $2 r=89 \mathrm{~mm}$ and a thickness of $0.15 \mathrm{~mm}$ glued on a spherically curved glass substrate with a radius of curvature of $R=860 \mathrm{~mm}$ [18]. This results in a captured solid angle of $\square 0.07 \mathrm{sr}$ or $1 / 180$ of the total $4 \pi \mathrm{sr}$. Crystal size and radius of curvature were chosen to provide the largest solid angle without significant loss in energy resolution through figure errors, stress and other geometrical contributions. Each crystal is aligned in a Johann geometry [19] consisting of the sample as the source, the analyzer and the detector on a Rowland circle of diameter $d=R$. 
Fig. 1a shows the schematic overall view of the spectrometer, and Fig. $1 \mathrm{~b}$ a more detailed view from the side and a view from the top.

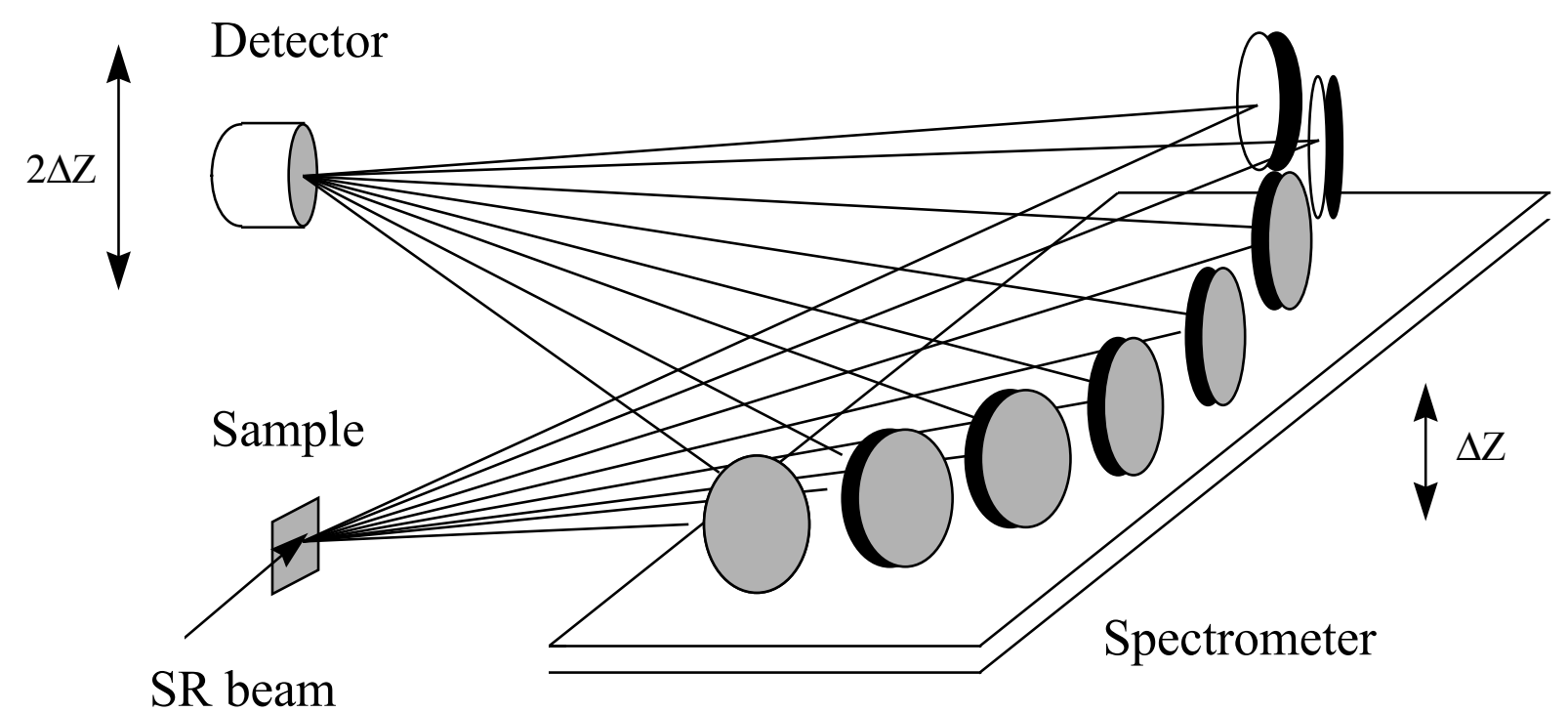

Figure 1a Schematic setup of the spectrometer geometry. All eight crystals are aligned to form identical symmetric triangles of source point, crystal and detector. Energy scans are performed by moving the spectrometer table and the detector (twice as much) in the vertical.

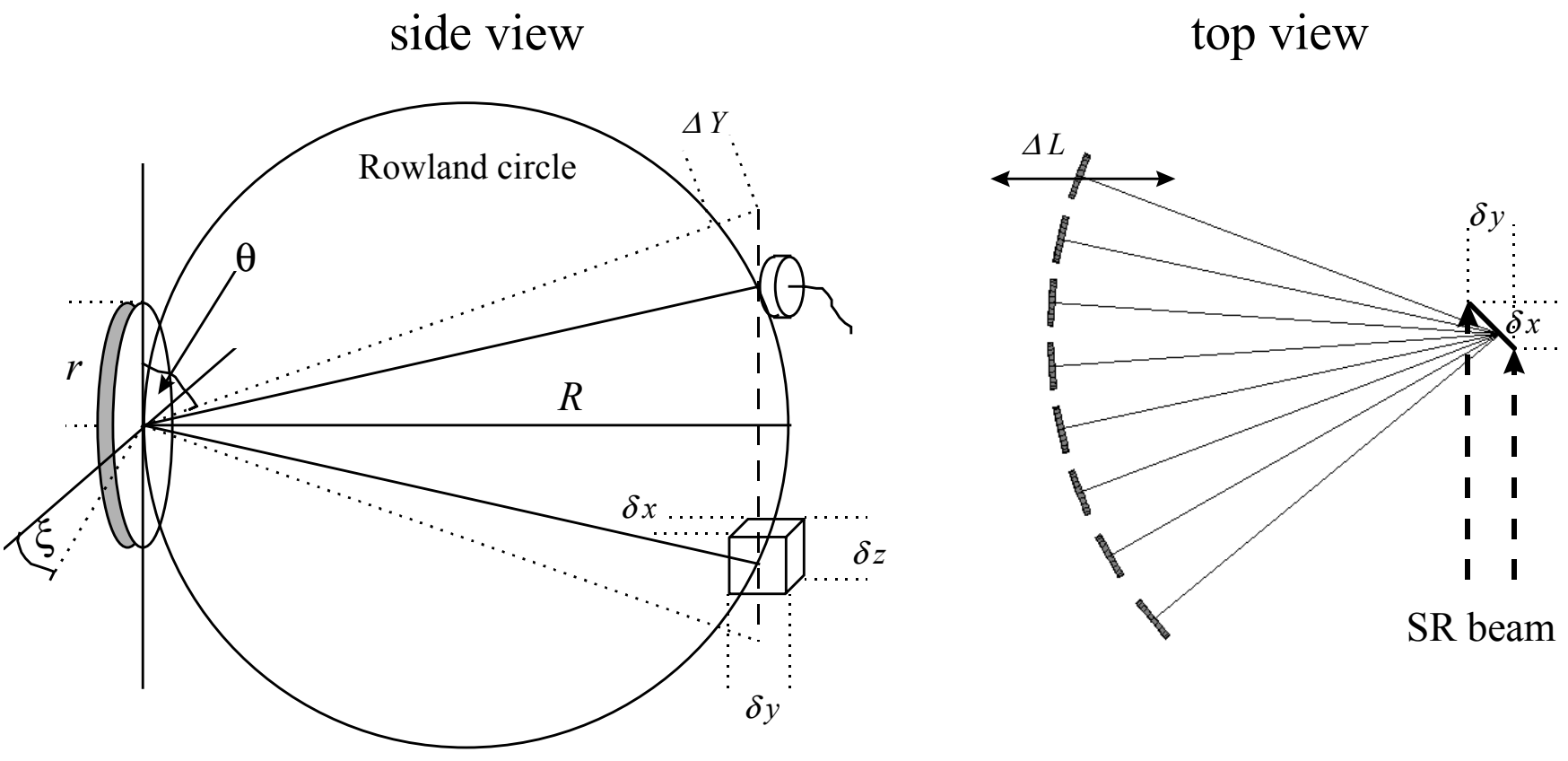

Figure 1b Left: Detailed side view of the Rowland geometry for a single crystal. Right: Top view of the schematic setup. Dimensions are arbitrarily scaled for better visualization.

In the left part of Fig. 1b the Johann geometry for an individual crystal is shown. To describe the performance of the whole instrument it is sufficient to discuss one crystal, since the geometry is from a spectroscopic point of view almost identical for each crystal. The top view in the right part of the figure shows that the center of the instrument (position between 
the fourth and fifth crystal) makes an angle towards the incident beam which is slightly smaller than $90^{\circ}$. This has been chosen as a compromise between larger background contributions at the crystals shown at the bottom and intensity losses at the crystals shown in the top resulting from a more grazing emission from the sample.

Let us discuss the different contributions which determine the instrumental energy resolution. First, there is the energy resolution resulting from the dynamical theory. For a $\mathrm{Si}(4,4,0)$ reflection this amounts to $\Delta E_{d y n}=62 \mathrm{meV}$ at an incident energy of $E_{0}=6.49 \mathrm{keV}$. Possible figure errors and d-spacing variations due to the bending are estimated to contribute $0.2 \mathrm{eV}$ resulting in a total intrinsic energy resolution of $\Delta E_{\text {int }} \Leftrightarrow 0.3 \mathrm{eV}$ [20]. Other geometrical contributions are discussed now in more detail (see also Fig. 1b):

1. Vertical source size: The vertical source size $\delta z$, which represents possibly the largest contribution to the energy resolution, results in an angular distribution $\Delta \theta=\delta z / R$ of the Bragg angle, and using Eq. (2) the energy contribution is given by:

$$
\Delta E_{z}=E(\delta z / R) \cot \theta
$$

2. Johann geometry: The fact that the diameter of the Rowland circle is identical to the radius of curvature of the crystal results for a Johann geometry (crystal planes are parallel to the crystal surface) in a slight deviation of the outer part of the crystal from the Rowland condition. Its effect is only significant in the vertical plane, resulting in an energy shift of the rays impinging on the crystal at a distance $r$ form the center given by:

$$
\Delta E_{J}=1 / 2 E(r / R)^{2} \cot ^{2} \theta
$$

3. Horizontal source size parallel to the crystal surface: The projection of the horizontal source size on the crystal plane, $\delta x$, contributes to a change of $\theta$ in second order. As can be easily shown for $\delta x<<2 R \cot \theta$ the energy contribution is given by:

$$
\Delta E_{x}=1 / 8 E(\delta x / R)^{2}
$$

4. Horizontal source size perpendicular to the crystal surface: The projection of the horizontal source size on the crystalnormal, $\delta y$, contributes to a change of $\theta$. For $\theta$ close to $90^{\circ}$ and $\delta y<<R$ this contribution is given by:

$$
\Delta E_{y}=E(\delta y / R) \cot ^{2} \theta
$$

5. Horizontal deviation from the Rowland condition: Suppose sample and detector are off the Rowland circle by $\Delta Y$ perpendicular to the crystal surface. Rays impinging on the crystal at radius $r$ from the center no longer form the same Bragg angle as rays impinging on the center. This difference in angle leads to an energy contribution given by:

$$
\Delta E_{Y}=E\left(r \Delta Y / R^{2}\right) \cot \theta
$$

6. Spherical bending: For any Bragg angle $\theta \neq 90^{\circ}$ the Rowland condition in a shperically bent crystal is only fulfilled in the vertical plane. Rays outside this plane impinge under a slightly different Bragg angle leading to an energy contribution given by:

$$
\Delta E_{S}=1 / 2 E(r / R)^{2} \cot ^{4} \theta
$$

Except for $\Delta E_{x}$ all the contributions are proportional to some order of $\cot \theta$ and diminish rapidly as $\theta$ approaches $90^{\circ}$. Furthermore, except for $\Delta E_{Y}$ and $\Delta E_{S}$, they are related to the source size and would disappear for a point source. Depending on the type of sample and the characteristics of the SR beam, it is, however, in general not possible to use a point like source. Biological samples, e.g., suffer from a fast photo reduction if the flux density of the SR beam becomes too large. The right choice of the source size is then a compromise of required energy resolution and acceptable sample damage. The energy contribution (1-5) can be combined to

$$
\Delta E_{\text {geo }}=\left[\left(\Delta E_{x}+0.75 \Delta E_{S}\right)^{2}+\left(\Delta E_{y}+0.87 \Delta E_{Y}\right)^{2}+\left(\Delta E_{z}+\Delta E_{J}\right)^{2}\right]^{1 / 2}
$$


where the three contributions under the square root are orthogonal. The factor 0.87 is a correction for the fact that the crystal is spherical and not quadratic and the factor 0.75 is the square of this since Eq. (7) is proportional to $r^{2}$. The total energy resolution $\Delta E_{\text {tot }}$ is obtained by a convolution of $\Delta E_{\text {int }}$ and $\Delta E_{\text {geo }}$. Since $\Delta E_{\text {int }}$ has approximately a Gaussian shape and $\Delta E_{\text {geo }}$ has approximately a square shape, the convolution results in a FWHM for $\Delta E_{\text {tot }}$ which is in between the sums and the square root of the square of the sums.

Let us know calculate $\Delta E_{\text {geo }}$ for different source sizes and compare results and experiment. The measured peak energy of $\mathrm{K} \beta$ in $\mathrm{MnF}_{2}$ is $\mathrm{E}_{0}=6492.7 \mathrm{eV}$ corresponding to a Bragg angle of $\theta=84.00^{\circ}$. We first assume that the spectrometer is exactly on the Rowland circle $\left(\Delta E_{Y}=0\right)$ and that the sample is illuminated by a source of $5 \mathrm{~mm}$ horizontal and a vertical beam dimension varying from $\delta z=0-2 \mathrm{~mm}$. The resulting source size for a sample tilted around a vertical axis under $45^{\circ}$ towards the incident beam direction is given by $\delta x=\delta y=5 \mathrm{~mm}, \delta z=0-2 \mathrm{~mm}$ (see also Fig. 1b). Using furthermore $r$ and $R$ as given above, the dependence $\Delta E_{\text {geo }}$ on $\delta z$ is shown in the left side of Fig. 2 compared with the calculation using $\delta x=\delta y=0$ (solid line).
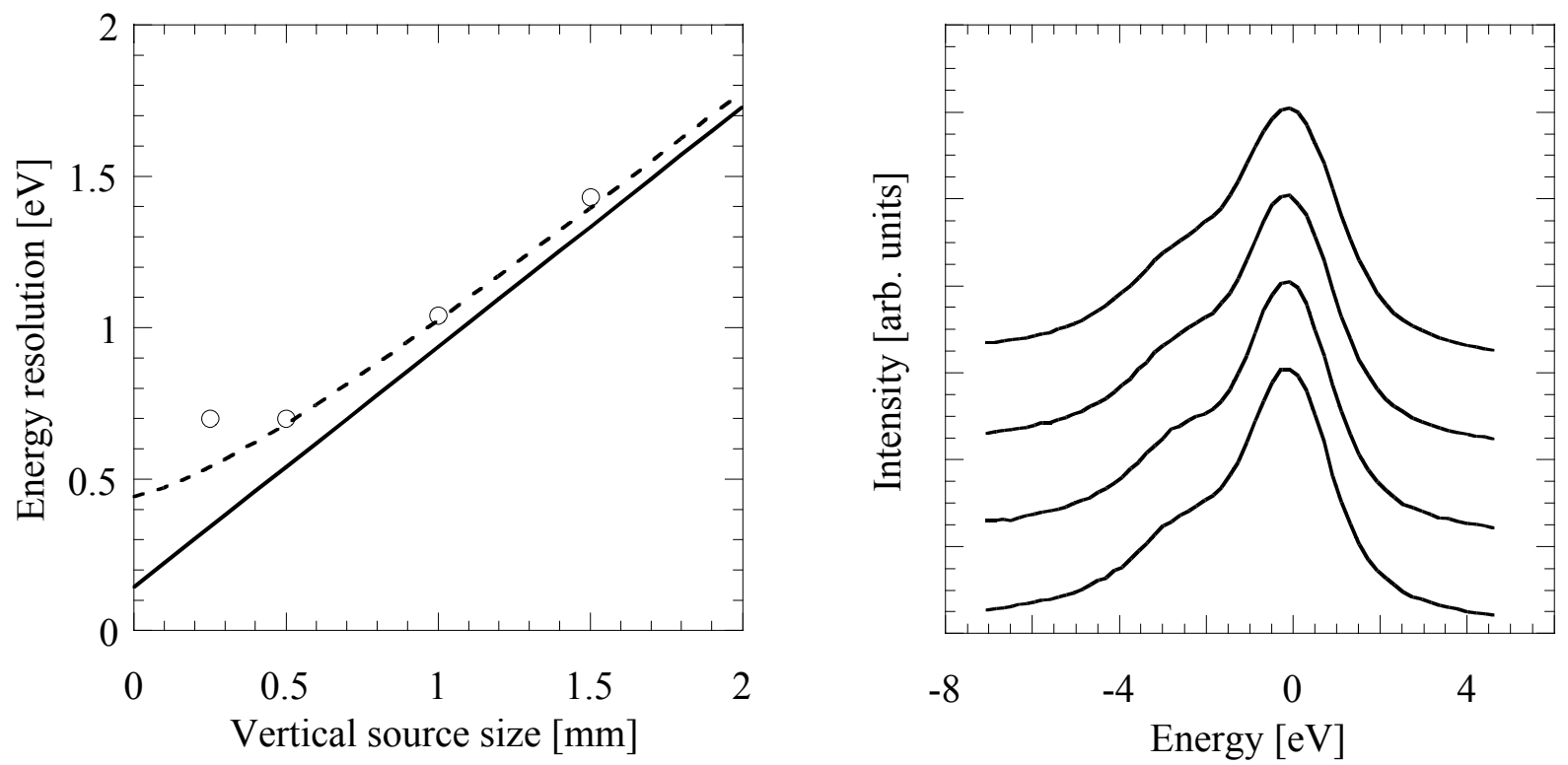

Figure 2 Left: Calculated geometrical energy contributions as a function of the vertical source size $\delta z$ for $\delta x=\delta y=5 \mathrm{~mm}$ (dashed line) and $\delta x=\delta y=0$ (solid line) compared with the measured total energy resolution (circles). Right: $\mathrm{K} \beta_{1,3}$ peak of $\mathrm{MnF}_{2}$ taken with $\delta x=\delta y=5 \mathrm{~mm}, \delta z=0.25,0.5,1.0,1.5 \mathrm{~mm}$ respectively (bottom to top).

For very small $\delta z$ the contribution of $\delta x$ and $\delta y$ amounts to $0.4 \mathrm{eV}$ but its relative contribution reduces to less than $0.1 \mathrm{eV}$ once $\delta z$ gets larger than $1 \mathrm{~mm}$. The figure clearly shows the importance of a small dz to achieve good energy resolution. In fact, the vertical scattering geometry was chosen due to the fact that most SR facilities provide smaller vertical than horizontal beam dimensions. The right graph of Fig. 2 shows spectra of the $\mathrm{K} \beta_{1,3}$ peak of $\mathrm{MnF}_{2}$ taken with $\delta x=\delta y=5 \mathrm{~mm}$ and $\delta z=0.25$, $0.5,1.0,1.5 \mathrm{~mm}$ respectively (bottom to top). The spectra show the majority spin shoulder [30] characteristic for high spin $\mathrm{Mn}^{+2}$, and the broadening with increasing $\delta z$ is apparent. The instrumental energy resolution can be obtained by deconvolution of the measured spectra and a resolution function which we assume to be Gaussian. As the reference spectra, we chose that taken by $\mathrm{H} 4 \mathrm{~m}(414$ inen et al. [20] with an energy resolution of $0.3 \mathrm{eV}$. As shown in Fig. 2 (left side) there is an excellent agreement with our calculated geometrical energy resolution down to $\delta z=0.5 \mathrm{~mm}$. At $\delta z=0.25 \mathrm{~mm}$ our measured resolution deviates from the calculation. This is, however, not surprising, since the calculation does not include the intrinsic resolution which we estimated to approximately $0.3 \mathrm{eV}$. 
A look on the $\mathrm{MnF}_{2}$ spectra shows that even a moderate energy resolution of $1-2 \mathrm{eV}$ is sufficient to obtain a good spectrum quality, since the intrinsic FWHM of Mn K $\beta$ fluorescence is of order 3 to $4 \mathrm{eV}$. Therefore $\mathrm{K} \beta$ emission spectroscopy can be performed with relatively large source sizes of order $1 \mathrm{~mm}$ vertical and $5 \mathrm{~mm}$ horizontal, whereas some of the other spectroscopies mentioned above much smaller source sizes.

\subsection{ALIGNMENT AND ENERGY SCANS}

An important practical characteristic of a multi crystal spectrometer is its simple tunability. We have changed this procedure from the previous designs by placing all the crystals on a spectrometer table which can be scanned vertically. Each crystal has three degrees of freedom for the individual alignment. There is a lateral adjustment $\Delta L$, to achieve the correct distance of the Rowland condition, and for the correct alignment of the crystal planes, the angles $\theta$ and $\xi$, can be varied (see Fig. 1b). After all crystals are aligned to form identical symmetric triangles of source, crystal and detector, energy scans are performed by vertical movement $\Delta Z$ of the spectrometer table. To track the reflected beam, any change $\Delta Z$ of the spectrometer table is accompanied by a change of $2 \Delta Z$ of the detector (see Fig. 1a). Besides the smaller susceptibility to errors of a two motor scan compared to a nine motor scan, this method has the advantage of keeping the geometry symmetric. If the energy were to be varied by simultaneously changing $\theta$ for all eight crystals and following with the detector but leaving the table fixed, the distance source-crystal would remain unchanged, but the distance crystal-detector would change, leading to a defocusing at the detector. Even though the effect on the energy resolution would be small, the defocusing of the reflected rays would require a much larger detector aperture and would consequently lead to more unwanted scattering background.

Some comments on our scan method are in place. First, it assumes that the fluorescence does not change with the emission angle. This assumption is easily justified considering that that the change in take-off angle is only a few degrees over an, e.g., $100 \mathrm{eV}$ scanning range and that furthermore excitations well above the K-edge results in isotropic X-ray fluorescence. Second, by vertically scanning crystals and detector, the Rowland condition is strictly fulfilled for only one energy (see left side of Fig. 1b). For any other Bragg angle $\Delta Y \neq 0$, leading to a contribution to $\Delta E_{\text {geo }}$ as given in Eq. (7). Fig. 3 shows the dependence on energy resolution considering the deviation from the Rowland condition for a long scan at the Mn K $\beta$ emission.

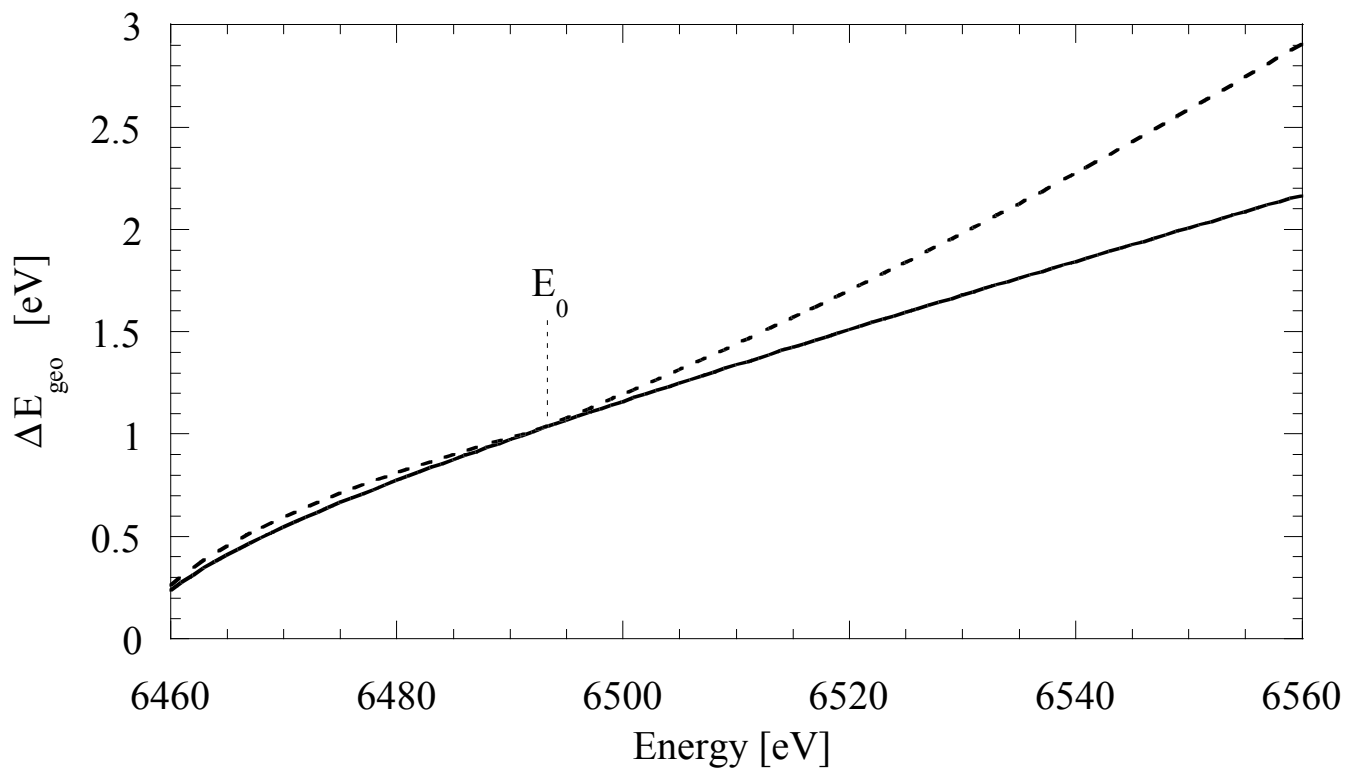

Figure 3 Calculated energy resolution for a long scan at the Mn K $\beta$ emission with (dashed line) and without (solid line) contributions resulting from the deviation of the Rowland condition in a vertical scan. The source size is set to $\delta x=\delta y=$ $5 \mathrm{~mm}, \delta z=1.0 \mathrm{~mm}$. 
The solid line is the calculation of $\Delta E_{\text {geo }}$ assuming $\delta x=\delta y=5 \mathrm{~mm}, \delta z=1.0 \mathrm{~mm}$ and $\Delta Y=0$. There is an approximately linear broadening of $\Delta E_{\text {geo }}$ as the Bragg angle decreases to $79.8^{\circ}$ at $6560 \mathrm{eV}$, reflecting the increasing source size contributions at decreasing Bragg angles. The dashed line shows the calculation including $\Delta Y$ resulting from a vertical spectrometer table scan, The correct Rowland condition is chosen to coincide with the $\mathrm{K} \beta_{1,3}$ peak energy $E_{0}$, where the two curves coincide. At lower energies the additional broadening is negligible but at larger energies the contributions start to increase linearly. It depends on the required energy resolution, if and when the crystal array has to be realigned to the correct Rowland condition during a long energy scan.

\subsection{INSTRUMENTAL DETAILS AND PERFORMANCE}

In this section the individual components of the instrument are briefly described. Besides the requirements of stability and reproducibility, other important issues such as minimization of intensity loss through absorption, minimization of unwanted background scattering and detector efficiency are addressed.

The spectrometer table is our own design and, to avoid angular instability during scans, it is driven by just one computer controlled stepper motor. A combination of three gear boxes distributes the rotational movement to four precision screws which are all aligned in phase leading to a lift of $1 \mathrm{~mm}$ per 2000 half steps, and we did not detect any angular tilt of the table during a $200 \mathrm{~mm}$ vertical scan. The table is designed to hold several thousand pounds of weight, which allows the extension to basically any realistic number of optical elements. All other motorized translational and rotational components are high precision devices from Huber X-ray diffraction equipment [21] driven by computer controlled stepper motors.

The motorized sample mount is designed to move heavy devices such as cryostats and allows vertical and horizontal alignment as well as rotation around the vertical axis. Typically a sample tilt of $45^{\circ}$ towards the direction of the incoming beam is chosen (see right side of Fig. 1b). It is important to note, that both horizontal and vertical movements are along the sample surface. This guarantees that any sample alignment does not change the source point with respect to the spectrometer, and thus the energy calibration. Small or non homogeneous samples can be optimized for the largest fluorescence signal, and samples sensitive to radiation damage can be easily illuminated at different positions without causing changes to the spectrometer calibration.

Stability and reproducibility are particularly important when a large number of individual scans are performed over long time periods. Ideally, the system should be decoupled from the experimental station such, that changes in beam position do not affect the energy calibration and can furthermore be corrected easily. To accomplish this important requirement the whole instrument including aperture slits, sample mount, detector mount and spectrometer table is placed on a large motorized optical table, which is usually part of the experimental station. The aperture slit, consisting of four motorized blades, defines the beam spot on the sample. An ion chamber is placed between aperture and sample to record the incident intensity $I_{0}$. Using a precision sight level, both detector and spectrometer table are aligned with respect to the beam spot on the sample, to define the symmetric triangle of source (beam spot), crystal and detector. Once this alignment is done scans are performed as described above. Suppose the SR beam moves either vertically or horizontally. Since the aperture is fixed with respect to the crystals, whatever is transmitted arrives at the correct position and hence does not cause any change in analyzer energy. Realignment to maximize $I_{0}$ is now easily performed by following the beam displacement with the optical table without affecting the energy calibration.

Air absorption in the path from the creation of the fluorescence (or scattered) photon in the sample to its final detection can be particularly large for lower energy X-rays. At $6.5 \mathrm{keV}$, e.g., the X-ray absorption length of air is $47 \mathrm{~cm}$ leading to an intensity loss of a factor 40 for a path length of $2 R=172 \mathrm{~cm}$. Several designs are possible. The cleanest solution, is to place the whole spectrometer inside a large vacuum tank with thin beryllium windows. Considering the complexity, weight and cost of such a tank makes this choice not very attractive for a spectrometer designed to be traveling between different beamlines. Previously there have been used other designs, such as rigid vacuum tanks with kapton windows and rigid He flight tubes. Both have the disadvantage of leaving a quite substantial air path which furthermore varies during a long scan. We decided therefore to use a flexible bag filled with helium. The material for the bag is commercially available 3 mil plastic, which has negligible absorption at $6.5 \mathrm{keV}$ and the helium itself transmits more than $99.7 \%$ in the $2 R$ path at this energy. The bag is connected to the rims of the analyzer crystals, the spectrometer table, the sample mount (or cryostat) and a mount just in front 
of the detector with reusable Valcro tape. This leaves an air path of less than $2 \mathrm{~cm}$. Due to the flexibility of the bag the air path is unchanged even when the instrument is scanned over a large range. The bag can be mounted in a matter of minutes and since it can be completely deflated before the filling, purging times are short, of order 30 minutes. Helium is continuously flowing from the top and exits through a small hole at the bottom. One standard 28 liter, 1800 psi bottle of helium lasts for more than one week.

To reduce the unwanted scattering background, the detector is lead shielded with an entrance aperture. Using a 1 x $3 \mathrm{~mm}^{2}$ source size, we measured a focal size of $1 \times 7 \mathrm{~mm}^{2}$ from all eight crystals combined. The horizontal broadening which results mostly from a parallax error in the alignment has essentially no influence on the energy resolution. To allow for slight defocusing during long scans we typically set our detector aperture to $3 \times 15 \mathrm{~mm}^{2}$. For dilute samples, which produce a very low fluorescence signal, a $\mathrm{LN}_{2}$ cooled Ge detector is used, for large signals from concentrated samples a standard NaI photomultiplier, or a photo diode can be utilized. All of these detectors have a quantum efficiency close to $100 \%$, however only the Ge detector has a good energy resolution. Even though the spectrometer discriminates against most of the background radiation, there is still a small fraction of elastic scattering and fluorescence from unwanted lines arriving at the detector. The good energy resolution of a Ge detector $(\mathrm{FWHM}=150 \mathrm{eV})$ allows to discriminate against these contributions and we measured a background signal of less than 1 photon/s for the very dilute photosystem II.

Finally, to demonstrate the performance of the spectrometer, Fig. 4 shows the $\mathrm{K} \beta$ spectrum of $\mathrm{MnF}_{2}$ on a logarithmic scale. The spectrum which was taken at the NSLS beamline X-25 shows a clean structure over several orders of magnitude, bringing out the much weaker features $\mathrm{K} \beta$ "' and $\mathrm{K} \beta_{2,5}$ at higher energies [22 -28]. Total scanning time was approximately 1 hour with five seconds per data point, at an incident intensity of $I_{0}=1.2 \times 10^{12}$ photons $/ \mathrm{s}$ using an aperture of $1 \times 2 \mathrm{~mm}^{2}$. To avoid detector saturation in the $\mathrm{K} \beta_{1,3}$ peak, $I_{0}$ was reduced for this region and the spectrum was rescaled. The peak counting rate corresponds to $6 \times 10^{5}$ photons/s, which is a factor 200 more than previously reported for a Fe $\mathrm{K} \beta$ spectrum (The fluorescence yield of $\mathrm{Fe}$ is approximately $30 \%$ larger than that of $\mathrm{Mn}$.) taken at similar incident flux [11]. From this comparison, it is clear that the instrument is capable to perform a qualitatively new type of spectroscopy.

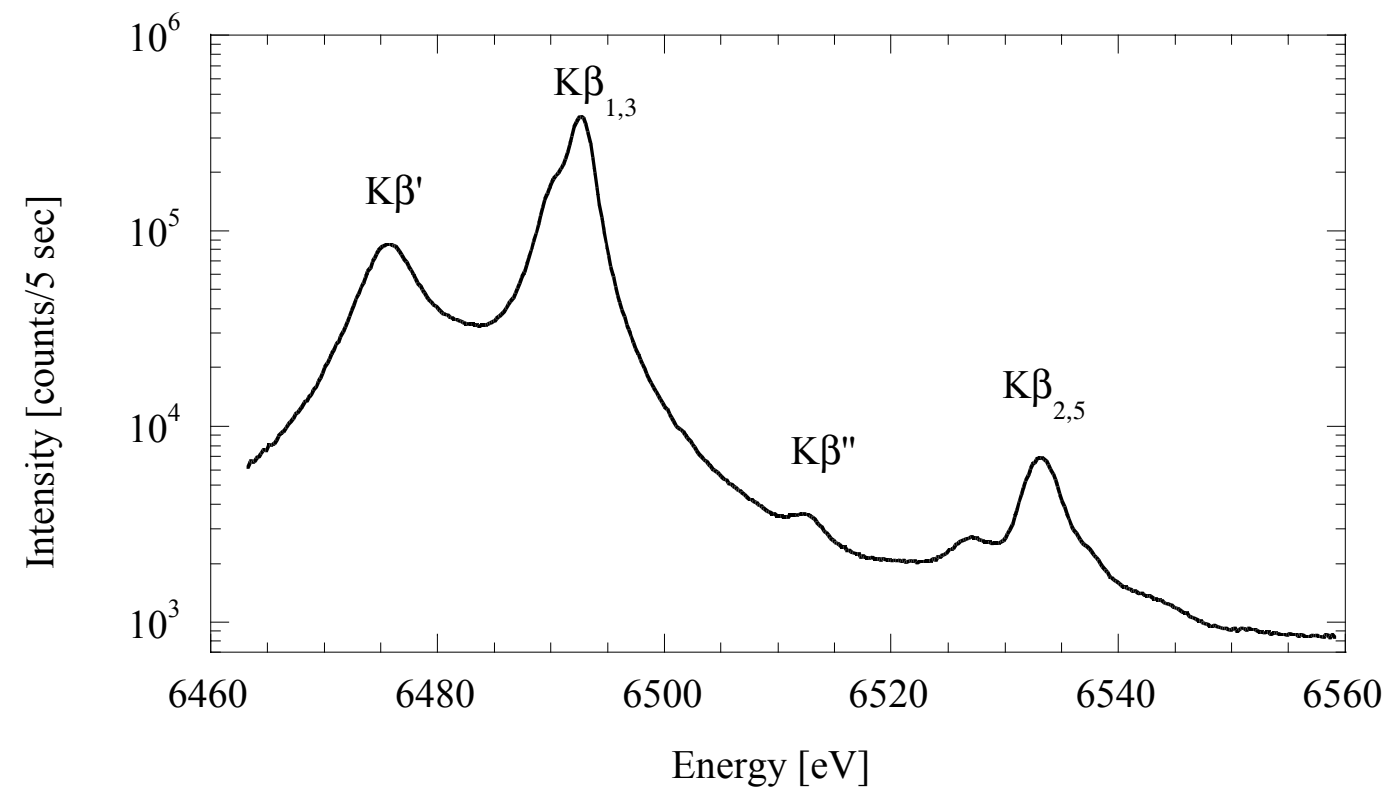

Figure $4 \mathrm{Mn} \mathrm{K} \beta$ spectrum of $\mathrm{MnF}_{2}$ taken at an incident flux of $1.210^{12}$ photons $/ \mathrm{sec}$.

\section{APPLICATIONS}

The original motivation for a high resolution large acceptance spectrometer as described above was the combined application of X-ray absorption and emission spectroscopy for dilute systems. However, any source of quasi isotropic X-rays, 
showing interesting features when analyzed with $1 \mathrm{eV}$ resolution can be the subject of study. To indicate the wide range of possible applications, two examples from very different fields are presented.

One example for an application in biochemistry is the Mn oxidation state assignment of the oxygen evolving complex in photosystem II, where traditionally other techniques like X-ray absorption or electron paramagnetic resonance are applied. Two different assignments for the Mn oxidations states of photosystem II in the S1 resting state have been proposed, Mn(III,III,III,III) and Mn(III,III,IV,IV) [31-35]. The left side of Fig. 5 shows Mn K $\beta$ spectra of Mn(II), Mn(III) and Mn(IV) models, displaying the typical shifts of the main peak to lower energies with increasing oxidation state [29,30]. The right side of Fig. 5 shows the Mn K $\beta$ spectrum of photosystem II in the S1 dark adapted state and its fit using a mixture of $50 \% \mathrm{Mn}(\mathrm{III})$ and $50 \% \mathrm{Mn}(\mathrm{IV})$ [36]. This result, combined with the assumption that photosystem II contains a $\mathrm{Mn}_{4}$ cluster favors the oxidation state assignment of Mn(III,III,IV,IV) over that of Mn(III,III,III,III). The Mn concentration in the sample was of order $200 \mathrm{ppm}$, and the spectrum was taken at SSRL beamline 6-2 with an incident flux of $10^{11}$ photons $/ \mathrm{sec}$.

Considering the fact that the fluorescence signal in photosystem II is three orders of magnitude lower than that of a concentrated sample, it is clear that only an efficient large acceptance spectrometer can obtain statistically significant results before photo reduction of the incident beam causes unacceptable sample damage.
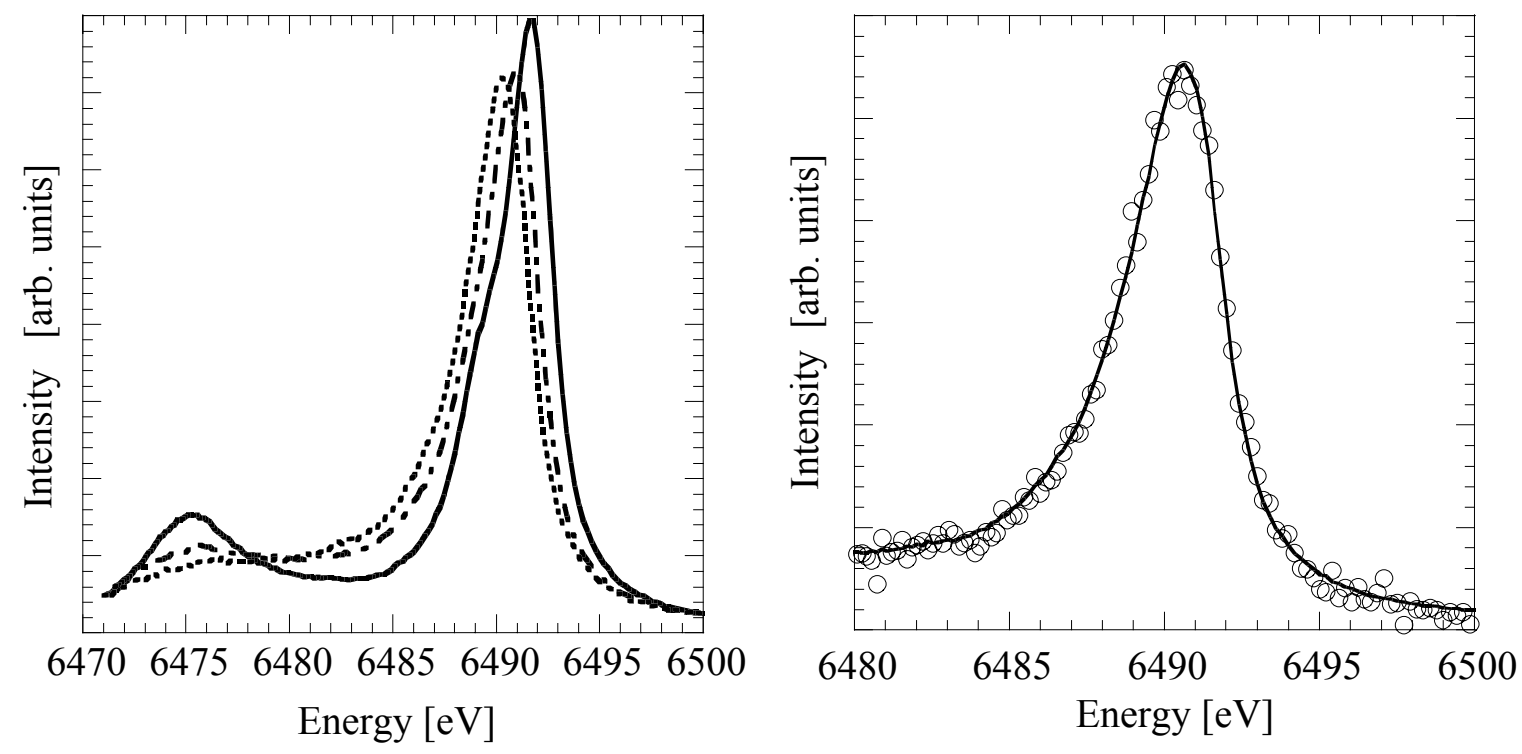

Figure 5 Left side: Mn K $\beta$ spectra of three model compounds for Mn(II) (solid line), Mn(III) (dash dotted line) and Mn(IV) (dotted line). Right side: Mn K $\beta$ main peak of photosystem II in the S1 resting state (circles) and best fit using a mixture of $50 \% \mathrm{Mn}(\mathrm{III})$ and $50 \% \mathrm{Mn}(\mathrm{IV})$

X-ray Raman spectroscopy is another technique which requires good energy resolution and a large solid angle. The spectrometer is tuned to a Bragg angle as close as possible to $90^{\circ}$ to guarantee the best energy resolution, and the incident energy $E$ is varied. Inelastically scattered photons can transfer their energy loss $E-E_{0}$ to a core level electron which is then promoted to an empty state [37-42]. Thus, in principle, Raman scattering gives the possibility to do soft X-ray absorption spectroscopy using hard X-rays. Since hard X-rays are a probe of the bulk properties, whereas soft X-rays interact at the surface, the two techniques complement each other for many systems. Furthermore, samples under extreme conditions, such as high pressure and/or temperature, as well as very reactive samples, can often not be studied with soft X-rays.

At the NSLS wiggler beamline X-25, we studied the carbon edge of graphite. Fig. 6 shows the comparison of our data with electron yield data obtained at the ALS [43]. Our combined energy resolution was $2 \mathrm{eV} \mathrm{FWHM,} \mathrm{at} \mathrm{an} \mathrm{incident} \mathrm{flux} \mathrm{of}$ $2 \times 10^{11}$ photons $/ \mathrm{sec}$. The scanning time for the spectrum was $20 \mathrm{sec}$ per data point and 2 hours total (the electron yield spectrum was taken with $15 \mathrm{sec}$ per point at an incident flux of $10^{11}$ photons $/ \mathrm{sec}$ ). Besides the differences attributed to the better energy resolution of the electron yield spectrum $(0.15 \mathrm{eV})$ there is a good agreement in the data. This demonstrates the 
feasibility of using the instrument for Raman spectroscopy. Employing our instrument at a third generation facility with better flux and energy resolution, Raman spectroscopy could become a routine technique for concentrated systems.

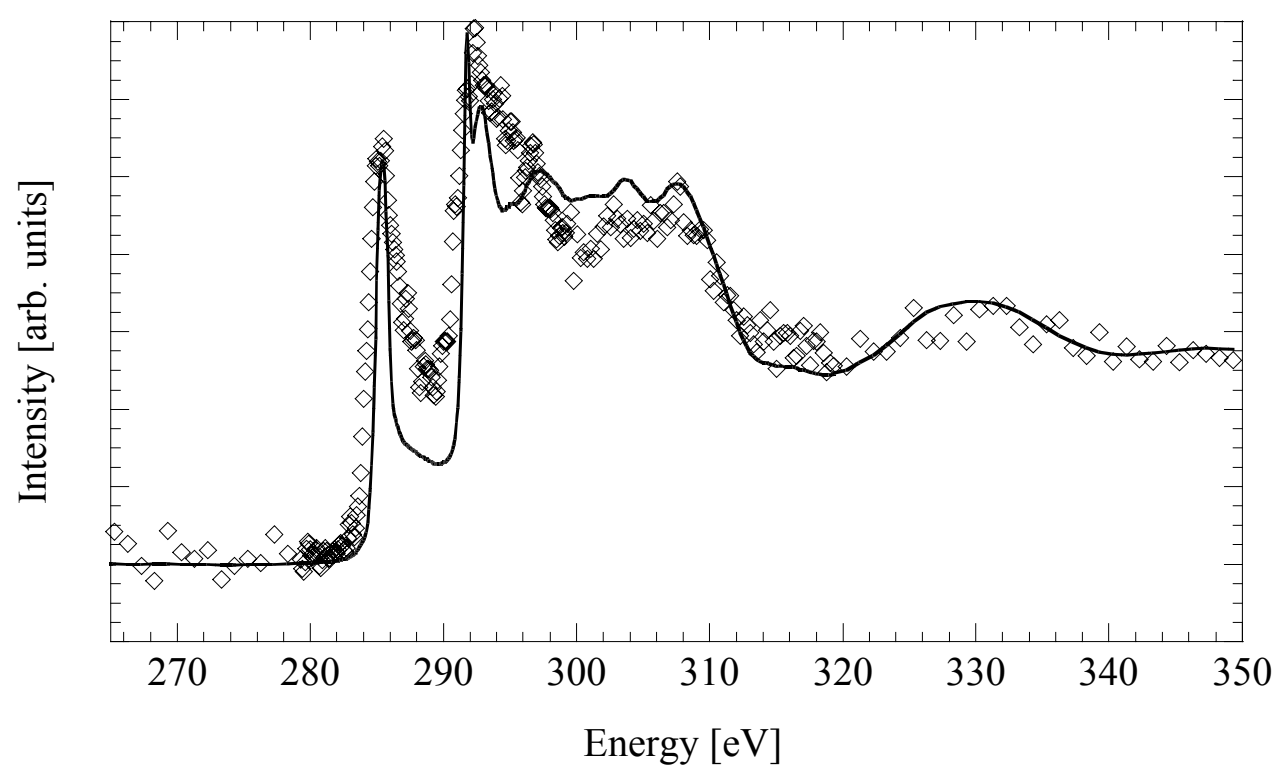

Figure 6 Comparison of the graphite K-edge spectra using electron yield (solid line) [44] and X-ray Raman spectroscopy (diamonds).

\section{Conclusions}

A newly designed multi crystal X-ray spectrometer has been described and characterized. The instrument, which can be applied in a large variety of fields such as X-ray absorption, emission, Raman and resonant Raman spectroscopy, employs 8 crystals of $89 \mathrm{~mm}$ diameter each, spherically bent to a radius of $86 \mathrm{~cm}$. The captured solid angle is $0.07 \mathrm{sr}$ with an energy resolution of order $1 \mathrm{eV}$, depending on the source size and Bragg angle. Different geometrical contributions to the energy resolution have been considered and compared with experimental results. The efficiency has been improved by more than two orders of magnitude compared to earlier results. Therefore the study of very dilute biological systems and/or very weak fluorescence and scattering signals has become possible, and two examples of such measurements are given. Finally, the instrument is designed to be very flexible; parameters such as scanning range, focusing length, and number of crystals can be easily varied. Essentially any set or combinations of crystals can be employed, individually or simultaneously.

\section{Acknowledgements}

The authors would like to thank the Drs. L. E. Berman and Z. Yin, at the NSLS as well as Dr. H. Tompkins and his group, at the SSRL. Dr. S. Anders is gratefully acknowledged for providing the graphite electron yield spectrum. The work on photosystem II is in collaboration with the Penner-Hahn group as well as Prof. C. F. Yochum at University of Michigan, Ann Arbor, the Christou Group at University of Indiana, Bloomington, and the Armstrong group at Boston College. The Raman work is done in collaboration with Dr. O. C. Mullins, Schlumberger-Doll Research Center, Ridgefield, CT. This work was funded by the National Institutes of Health (GM-44380 and GM-48145) and by the Department of Energy, Office of Biological and Environmental Research. The Stanford Synchrotron Radiation Laboratory and the National Synchrotron Light Source are supported by the Department of Energy, Office of Basic Energy Sciences. 


\section{References}

1. For a review of the early history of synchrotron radiation see: Winick and S. Doniach, "Synchrotron Radiation Research", Plenum Press: New York, 1980.

2. T. 4 berg and J. Tulkki, “Atomic Inner-Shell Physics”, edited by B. Crasemann, Plenum Press: New York, 1985.

3. P. L. Cowan, Resonant Anomalous X-ray Scattering, edited by G. Materlik, C. J. Sparks and K. Fischer, pp. 449-472, North-Holland: Amsterdam, 1994.

4. A. Mori, Y. Kayanuma and A. Kotani, Prog. Theor. Phys. 106, pp. 75-93, 1991.

5. P. Skytt, J. H. Guo, N. Wasserdahl and J. Nordgren, Phys. Rev. A, 52, pp. 3572-3576, 1995.

6. F. M. F. deGroot, Phys. Rev. B, 53, pp. 7099-7110, 1996.

7. M. H. Krisch, C. C. Kao, F. Sette, W. A. Caliebe, K. H®m®14inen and J. B. Hastings, Phys. Rev. Lett., 74, pp. 49314934, 1995.

8. P. Carra, M. Fabrizio and B. T. Thole, Phys. Rev. Lett., 74, pp. 3700-3703, 1995.

9. Y. Ugadawa, H. Hayashi, K. Tohji and T. Mizushima, J. Phys. Soc. Jpn., 63 pp. 17130-1720, 1994.

10. V. Stojanoff, K. H(4m(414inen, D. P. Siddons, J. B. Hastings, L. E. Berman, S. P. Cramer and G. Smith, Rev. Sci. Instrum., 63, pp. 1125-1127, 1992.

11. X. Wang, M. M. Grush, A. G. Froeschner and S. P. Cramer, J. Synchrotron Rad., 4, pp. 236-242, 1996.

12. W. H. Zachariasen, "Theory of X-Ray Diffraction in Crystals", John Wiley and Sons, Inc. 1945.

13. R. W. James, "Optical Principles of the Diffraction of X-Rays", G. Bell and Sons, 1950.

14. R. W. James, "The dynamical theory of x-ray diffraction, Solid State Physics, 15, pp. 53, 1963.

15. B. W. Batterman and H. Cole, "Dynamical diffraction of x-rays by perfect crystals", Rev. Mod. Phys., 36, pp. 681-717, 1964.

16. J. W. M. DuMond, Phys. Rev. 52, pp. 872-883, 1937.

17. C. Masciovecchio, U. Bergmann, M. Krisch, G. Ruocco, F. Sette and R. Verbeni, “'A perfect crystal X-ray analyser with meV energy resolution"', Nucl. Instrum. Methods, B, 111, pp. 181-186, 1996.

18. the crystals were purchased from Crismatec, B. P. 521, 77794 Nemours Cedex, France, e-mail: crismatecoptic@calvanet.calvacom.fr.

19. H. H. Johann, Z. Physik, 69, pp. 185, 1931.

20. K. H(4m(4)14inen, C.-C. Kao, J. B. Hastings, D. P. Siddons, L. E. Berman, V. Stojanoff and S. P. Cramer, Phys. Rev. B, 46, pp. 14274-14277, 1992.

21. Huber Diffraktionstechnik GmbH, Sommerstr,. 4, D-83253 Rimsting, Germany, Tel.: (49) 8051-4472, Fax: (49) 805161680

22. P. E. Best, Bull. Am. Phys. Soc. 10, pp. 29, 1965.

23. P. E. Best, J. Chem. Phys., 44, pp. 3248-3253, 1966.

24. A. S. Koster and H. Mendel, J. Phys. Chem. Solids, 31, pp. 2511-2522, 1970.

25. D.S. Urch, X-ray Emission Spectroscopy, chapter in: Electron Spectroscopy: Theory, Techniques and Applications, vol. 3, edited by: Brundle, C. R.; London, A. D., UK: Academic Press, pp. 1-39, 1979.

26. J. B. Jones and D. S. Urch, J. C. S. Dalton, pp. 1885, 1975.

27. K. Tsutsumi, H. Namori, and K. Ichikawa, Phys. Rev. B, 13, pp. 929-933, 1976.

28. U. Bergmann, C. R. Horne, T. J. Collins and S. P. Cramer, in progress.

29. K. Tsutsumi, , J. Phys. Soc. Jpn., 14 pp. 1696, 1959.

30. G. Peng, F. M. F. deGroot, K. H(4) (414inen, J. A. Moore, X. Wang, M. M. Grush, J. B. Hastings, D. P. Siddons, W. H. Armstrong, O. C. Mullins and S. P. Cramer, J. Am. Chem. Soc., 116, pp. 2914-2920, 1994.

31. R. J. Debus, Biochimica Et Biophysica Acta, 1102, pp. 2691992.

32. M. Zheng M.; G. C. Dismukes, Inorg. Chem., 35, pp. 3307, 1996.

33. V. K. Yachandra, K. Sauer, M. P. Klein, Chem. Rev., 96, pp. 2927-2950, 1996.

34. P. J. Riggs, R. Mei, C. F. Yocum, J. E. Penner-Hahn, J. Am. Chem. Soc., 114, pp. 10650, 1992.

35. M. Kusunoki,, T. Ono, T. Matsushita, H. Oyanagi, Y. M. N. Inoue, J. Biochem., 108, pp. 560, 1990.

36. U. Bergmann, M. M. Grush, C. R. Horne, P. DeMarois, J. E. Penner-Hahn, C.F. Yocum, D.W. Wright, C .E. Dub(2), W. H. Armstrong, G. Christou, H. J. Eppley and S. P. Cramer, J. Phys. Chem., submitted.

37. T. Suzuki, J. Phys. Soc. Jpn., 22, pp. 1139, 1967.

38. Y. Mizuno and Y. Ohmura, J. Phys. Soc. Jpn., 22, pp. 445, 1967.

39. K. Tohji ans Y. Ugadawa, Phys. Rev. B, 36, pp. 9410, 1987.

40. K. Tohji ans Y. Ugadawa, Phys. Rev. B, 39, pp. 7590, 1989. 
41. W. Schulke, U. Bonse, H. Nagasawa, A. Kaprolat and A. Berthold, Phys. Rev. B, 38, pp. $2112,1998$.

42. K. Tohji ans Y. Ugadawa, T. Matsushita, M. Nomura and T. Ishikawa, J. Chem. Phys., 92, pp. $3233,1990$.

43. S. Anders, J. Diaz, J. W. Ager III, R. Y. Lo, and D. B. Bogy, Appl. Phys. Lett. 7, pp. 3367-3369, 1997. 